\title{
AMMONIA CLUMPS IN THE ORION AND CEPHEUS CLOUDS
}

\author{
J. HARJU ${ }^{1}$, C.M. WALMSLEY ${ }^{2}$ AND J.G.A. WOUTERLOOT ${ }^{3}$ \\ ${ }^{1}$ Observatory and Astrophysics Laboratory, University of Helsinki \\ ${ }^{2}$ Max-Planck-Institut für Radioastronomie, Bonn \\ ${ }^{3}$ I. Physikalisches Institut, Universität zu Köln
}

\begin{abstract}
We present statistics of clump properties in the Orion and Cepheus cloud complexes based on ammonia mapping observations. Surroundings of about 50 IRAS sources earlier found to have associated molecular line emission (Wouterloot, Walmsley and Henkel, 1988) were mapped in NH3 $(1,1)$ and $(2,2)$ with the Effelsberg 100 -m telescope. Our main interest has been in determining the clump sizes and masses on the basis of the ammonia column density distribution, which together with the observed velocity dispersion lead to a rough estimate of the dynamical state. We also have studied the starclump separations which should give us estimates of the source ages. Special attention has been paid to comparison of our Orion data with the Benson and Myers (1989, hereafter BM89) results in Taurus because the linear resolutions in the two studies are similar.
\end{abstract}

Molecular clouds contain cool, high density cores, which are often associated with newly born stars detected in infrared surveys - above all IRAS. This has become evident through the work of Myers, Benson and collaborators in nearby molecular cores (see e.g. Beichman et al. 1986, Myers et al. 1987, BM89), and the surveys by Wouterloot, Walmsley and Henkel in $\mathrm{H}_{2} \mathrm{O}$ maser, $\mathrm{NH}_{3}$ and $\mathrm{CO}$ lines towards IRAS point sources in Orion and Cepheus (Wouterloot et al. 1986, 1988, 1989).

We have studied the clump properties, such as sizes, masses, temperatures and densities in the Orion and Cepheus giant molecular cloud complexes towards sources detected by Wouterloot et al. (1988). The observations were made with the 100-m telescope in Effelsberg by measuring the $\mathrm{NH}_{3}(1,1)$ and $(2,2)$ transitions. Our Orion sample (L1630 and L1641) consists of 24 separate clumps in the neighbourhood of 16 IRAS point sources. In the Cepheus region, the surroundings of 29 IRAS sources were mapped. A summary of the statistical results of this study is given in the attached table.

The Orion clumps are larger, warmer, more massive and have broader lines than the Taurus clumps of BM89. Despite their relatively large linewidths, most Orion clumps seem to be gravitationally bound. This fits the concept of gravitationally dominated collapse in high-mass star forming regions (Shu et al. 1987), and is consistent with the NIR observations of Strom et al. (1989a,b,c) towards L1641, where they found a large number of sources embedded in high-opacity cores. 8 of their sources are located inside clumps mapped by us.

In Cepheus, the derived densities are lower than in Orion or Taurus. Especially in the direction of the Cepheus OB3 cloud (adopted distance $730 \mathrm{pc}$ ), the cores seem to lack mass to balance their "turbulent" motions. The observed densities are, however, likely to be affected by beam dilution. Furthermore, the uncertainty of the distance makes the mass estimates less reliable.

One distinctive property of Orion is that the embedded stars usually lie very 
close to the clump centers, in a small range below $0.1 \mathrm{pc}$ which is about a typical clump radius, while in the cases of the other complexes the distribution is broader. If we interpret this star-clump separation as measuring the age of the star, the implication is that most embedded stars detected as IRAS point sources in Orion were born during a short period about $10^{5}$ years ago.

Table. Average clump properties in Orion, Cepheus and Taurus. Columns : 1) infrared luminosity of the IRAS source $\left(\mathrm{L}_{\odot}\right) ; 2$ ) clump mass $\left(\mathrm{M}_{\odot}\right) ; 3$ ) clump half power diameter (pc); 4) clump-star distance (pc); 5) $\mathrm{NH}_{3}(1,1)$ line width $\left.\left(\mathrm{kms}^{-1}\right) ; 6\right)$ kinetic temperature $(\mathrm{K})$; $\mathrm{i})$ maximum excitatation temperature $(\mathrm{K})$; 8) maximum $\mathrm{H}_{2}$ density $\left(\mathrm{cm}^{-3}\right)$; 9 ) maximum $\mathrm{NH}_{3}$ column density $\left.\left(\mathrm{cm}^{-2}\right) ; 10\right)$ number of separate clumps included in the statistics. Notes : 1) STD means standard deviation. 2) The densities are calculated using the two-level approximation formula of Ho and Townes (1983) on the basis of the derived kinetic and excitation temperatures. For comparison, the densities derived by BM89 based on an LVG analysis are tabulated with emphasized numbers.

\begin{tabular}{|c|c|c|c|c|c|c|c|c|c|c|c|}
\hline & & $\begin{array}{l}L_{m} \\
\left(L_{0}\right)\end{array}$ & $\begin{array}{c}\mathbf{M} \\
\left(\mathbf{M}_{\boldsymbol{\theta}}\right)\end{array}$ & $\begin{array}{l}\text { diem } \\
\text { (pe) }\end{array}$ & (pe) & $\begin{array}{l}\langle\Delta v\rangle \\
(1+\infty-1)\end{array}$ & $\begin{array}{c}\left\langle T_{\text {tion }}>\right. \\
\text { (K) }\end{array}$ & $\begin{array}{c}\max \left(T_{e e}\right) \\
\text { (K) }\end{array}$ & $\begin{array}{c}\max \left(\mathrm{n}_{\mathrm{m}_{2}}\right) \\
\left(.10^{4} \mathrm{~cm}^{-3}\right)\end{array}$ & $\begin{array}{c}\max (N)) \\
\left(\cdot 10^{d 4} \mathrm{~cm}^{-2}\right)\end{array}$ & sample \\
\hline $\begin{array}{c}\text { Orton } \\
\text { diet } \sim 500 \text { pe }\end{array}$ & $\begin{array}{l}\text { average } \\
\text { STD } \\
\text { median }\end{array}$ & $\begin{array}{c}50 \\
(52) \\
28\end{array}$ & $\begin{array}{c}26 \\
(22) \\
16\end{array}$ & $\begin{array}{l}0.18 \\
(0.06) \\
0.17\end{array}$ & $\begin{array}{l}0.08 \\
(0.05) \\
0.08\end{array}$ & $\begin{array}{c}0.81 \\
(0.28) \\
0.78\end{array}$ & $\begin{array}{l}18.7 \\
(4.6) \\
14.7\end{array}$ & $\begin{array}{c}9.2 \\
(2.8) \\
8.8\end{array}$ & $\begin{array}{l}10.0 \\
(9.4) \\
9.2\end{array}$ & $\begin{array}{r}7.7 \\
(4.7) \\
6.6\end{array}$ & 24 \\
\hline $\begin{array}{l}\text { Cepheus (neas) } \\
\text { dist } \sim 730 \text { pe }\end{array}$ & $\begin{array}{l}\text { sverase } \\
\text { STD } \\
\text { medien }\end{array}$ & $\begin{array}{c}110 \\
(130) \\
55\end{array}$ & $\begin{array}{l}16 \\
(11) \\
15\end{array}$ & $\begin{array}{c}0.18 \\
(0.10) \\
0.17\end{array}$ & $\begin{array}{l}0.11 \\
(0.10) \\
0.07\end{array}$ & $\begin{array}{c}1.0 \\
(0.2) \\
1.0\end{array}$ & $\begin{array}{l}16.7 \\
(1.8) \\
17.0\end{array}$ & $\begin{array}{l}5.0 \\
(1.6) \\
6.0\end{array}$ & $\begin{array}{l}2.1 \\
(1.4) \\
2.1\end{array}$ & $\begin{array}{r}4.6 \\
(3.0) \\
3.3\end{array}$ & 11 \\
\hline $\begin{array}{l}\text { Cephens (far) } \\
\text { dist } \sim 3500 \text { pe }\end{array}$ & $\begin{array}{l}\text { orerage } \\
\text { STD } \\
\text { median }\end{array}$ & $\begin{array}{l}2.5(4) \\
(3.0(4)) \\
1.0(4)\end{array}$ & $\begin{array}{l}590 \\
(910) \\
140\end{array}$ & $\begin{array}{c}0.98 \\
(0.38) \\
0.80\end{array}$ & $\begin{array}{c}0.83 \\
(0.23) \\
0.56\end{array}$ & $\begin{array}{l}1.5 \\
(0.4) \\
1.5\end{array}$ & $\begin{array}{l}20.4 \\
(3.7) \\
10.7\end{array}$ & $\begin{array}{l}5.7 \\
(1.7) \\
5.7\end{array}$ & $\begin{array}{l}1.9 \\
(1.4) \\
1.7\end{array}$ & $\begin{array}{r}4.7 \\
(5.3) \\
2.2\end{array}$ & 15 \\
\hline $\begin{array}{c}\text { Taurus } \\
\text { dist } \sim 140 \text { pe } \\
(B M 80)\end{array}$ & $\begin{array}{l}\text { everese } \\
\text { STD } \\
\text { modiea }\end{array}$ & $\begin{array}{l}1.1 \\
(1.4) \\
0.6\end{array}$ & $\begin{array}{c}9 \\
(11) \\
4\end{array}$ & $\begin{array}{c}0.10 \\
(0.04) \\
0.08\end{array}$ & $\begin{array}{c}0.13 \\
(0.10) \\
0.12\end{array}$ & $\begin{array}{c}0.30 \\
(0.07) \\
0.28\end{array}$ & $\begin{array}{l}10.0 \\
(2.1) \\
9.6\end{array}$ & $\begin{array}{l}7.6 \\
(1.6) \\
7.6\end{array}$ & 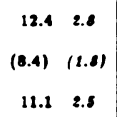 & $\begin{array}{l}9.6 \\
(4.5) \\
10.0\end{array}$ & 10 \\
\hline notes & 11 & & & & & & & & 2) & & \\
\hline
\end{tabular}

\section{References}

C.A. Beichman, P.C. Myers, J.P. Emerson, S. Harris, R. Mathieu, P.J. Benson, R.E. Jennings : 1986 Astrophys. J., 307, 337

P.J. Benson, P.C. Myers : 1989 Astrophys. J. Suppl., 71, 89 (BM89)

Y. Fukui : 1989 in "Low Mass Star Formation and Pre-Main-Sequence Objects" (ESO

Workshop, Garching 11-13 July 1989, Proceedings edited by B. Reipurth)

P.T.P Ho, C.H. Townes : 1983 Ann. Rev. Astron. Astrophys., 21, 239

P.C. Myers, G.A. Fuller, R.D. Mathieu, C.A. Beichman. P.J. Benson, R.E. Schild, J.P. Emerson : 1987 Astrophys. J., 319, 340

F.H. Shu, F.C. Adams, S. Lizano : 1987 Ann. Rev. Astron. Astrophys., 25, 23

K.M. Strom, G. Newton, S.E. Strom, R.L. Seaman, L. Carrasco, I. Cruz-Gonzales, A.

Serrano, G.L. Grasdalen : 1989a Astrophys. J. Suppl., 71, 183

K.M. Strom, M. Margulis, S.E. Strom : 1989b Astrophys. J., 345. Li9

K.M. Strom, M. Margulis, S.E. Strom : 1989c Astrophys. J., 346, L33

J.G.A. Wouterloot, C.M. Walmsley : 1986 Astron. Astrophys., 168, 237

J.G.A. Wouterloot, C.M. Walmsley, C. Henkel : 1988 Astron. Astrophys., 203, 367

J.G.A. Wouterloot, C. Henkel, C.M. Walmsley : 1989 Astron. Astrophys., 215, 131 